\title{
Helices with optical activity
}

\section{A neat conformation of a neat prediction points to more problems in the interpretation of quantum mechanics. In what direction do we turn now?}

THE complexities of quantum mechanics persist not merely at the cutting edge represented by particle physics, but in much more elementary manifestations. It is a curious state of affairs. Students are taught how to calculate the wave function of, say, the state of an electron, but many go on to spend their lives worrying about quantum chromodynamics, string theory and the like; yet quite simple questions remain unanswered. Why, for example, is it supposed that the square of the amplitude of a wave function represents the probability distribution of the electron? It is justifiable chiefly because it works. Now there is a rush of interest in a set of problems springing from another longrecognized ambiguity in the calculation of a wave function, that is whether the phase of the result has physical significance of any kind. One of the most recent developments appears to vindicate an important prediction (Proc.Roy.Soc. 45, 1984) by Dr M.V. Berry at the University of Bristol.

The problem is usually well taught to students. The solution of, say, a Schrödinger wave-equation for an electron in a time-independent electrical potential is a function which is, in general, algebraically complex: the value of the function at any point is a complex number, with a real and an imaginary part. The obvious physical correlate of this result is the probability that the electron will turn up at that point, which is simply the square of the amplitude of the complex number, or the sum of the squares of its real and imaginary parts. In that representation of complex numbers by a point in the two-dimensional plane in which the real and imaginary parts are the displacements from the origin along two perpendicular axes, the amplitude is the length of the radius vector to the representative point. The phase, the angle whose tangent is the ratio of the displacement along the two axes, appears to have no physical significance.

All representations of quantum mechanics have the same property. Whether the states of systems are represented by functions (Schrödinger), vectors (Heisenberg) or elements in a formal algebra (Dirac), all these quantities may be multiplied by complex numbers (or functions) chosen arbitrarily except for the condition that their amplitude is always exactly unity without the calculation of the usual physical quantities being affected in any way. So is the phase entirely without physical significance? And if so, why cannot quantum mechanics be constructed so as to dispense with it?

People agreed on this until about twenty years ago, extrapolating from the Aharonov-Bohm effect. While the phase of a wave function representing the state of some system may be irrelevant in the calculation of measurable quantities such as the total energy, the place to look for the effects attributable to phase is among interference phenomena, for which purpose only changes of phase can matter. There are circumstances in which the phase of, say, an electron wave-function, however arbitrary or irrelevant, may be made to vary systematically. Magnetic fields (which may deflect electrons while doing no work on them) may do the trick; there have recently been at least two demonstrations of interference between the two halves of a split electron beam whose phases have been changed differently by magnetic fields.

Berry's argument is a generalization of the Aharonov-Bohn effect. The properties of a quantum state may potentially be determined by many parameters; an externally applied magnetic field is merely one. Each parameter will appear in the Hamiltonian representing the energy of the system as a whole, to each value of each of which corresponds a different set of stationary states. It is possible to imagine that one of these parameters is altered systematically, but so slowly that a stationary state becomes another (adiabatic is the word), and in a way that returns it to its starting-point. Physically, the system will then be back where it started. Will the phase of the wave function have been changed? Berry says yes, in general.

Recent intriguing evidence bears him out. The physical system is the simplest possible, the polarized photon, characterized by just two dynamical variables; the wave vector (which must lie in the direction of propagation) and the sense of polarization (which may be left-handed or right-handed, viewed along the direction of propagation). Raymond Y. Chiao from the University of California at Berkeley and Yong-Shi Wu from the University of Utah now point out (Phys. Rev. Lett. 57, $933 ; 1986)$ that, for circularly polarized photons in, say, an optical fibre, the propagation vector, is simply the direction of travel, is determined by the shape of the track followed by the fibre, and thus is a parameter in Berry's sense. If the fibre is not too sharply kinked, photons will remain polarized with sense unchanged.

What happens to the phase? It depends on the contortions of the propagation vector as the photon travels along the fibre. Chiao and $\mathrm{Wu}$ infer from Berry's work that two fibres of the same optical path length wound into helices which have opposite senses will have equal but opposite effects on the phase of the quantum state of circularly polarized photons. So why not take a single beam of photons, split it into two, direct each half into oppositely wound helical fibres and look for destructive interference? The difficulty is that of producing left and right-handed helices which are exact mirror imges. Chiao and Akira Tomita (working then at AT\&T Bell Laboratories) have instead measured the optical rotation that occurs when a linearly polarised beam of photons traverses an optical fibre wound in helices of different pitch (Phys. Rev. Lett. 57, 937 ; 1986). Inevitably, Berry's prediction is confirmed.

Berry's argument goes to the root of a string of deep questions in quantum mechanics. There are obvious connections with the way in which the magnetic flux enclosed by a superconducting loop, a Josephson junction for example, is quantized. There are connections with Dirac's famous magnetic monopoles. A feature of Berry's construction is that the change of phase appears to be determined only by geometrical or, strictly, topological considerations. The phase shift generated by a single turn of a uniform helix is determined only by the path traced out on the surface of a sphere by the changing propagation vector. For a helix, this path is a circle, but it emerges that any other closed path (meaning any other fibre path) would have the same effect on the phase so long as the wave-vector traces out a closed loop enclosing a patch of surface on the same hypothetical sphere which subtends the same solid angle at the origin.

The other side of the coin is that both authors remark that their experimental results are those that would have been obtained classically, by using Maxwell's equations to calculate the intrinsic optical activity of a helically wound fibre. The novelty, not yet tested, is that Berry's result should obtain even with single photons. If that prediction prove correct it points to a novel correspondence between classical and quantum mechanics.

John Maddox 